\title{
Brain cell membrane motion-restricted phospholipids in patients with schizophrenia who have seriously and dangerously violently offended
}

\author{
Basant K Puri ${ }^{1}$, Serena J Counsell', Gavin Hamilton², Marcelo G Bustos ${ }^{3}$, lan H Treasaden ${ }^{4 *}$ \\ From $1^{\text {st }}$ International Congress on Neurobiology and Clinical Psychopharmacology \\ and European Psychiatric Association Conference on Treatment Guidance \\ Thessaloniki, Greece. 19-22 November 2009
}

\section{Background}

This study directly assessed, for the first time, whether, as expected under the membrane phospholipid hypothesis of schizophrenia, there was a change in brain cell motion-restricted membrane phospholipids in vivo in male forensic patients with schizophrenia who had seriously and violently offended (homicide, attempted murder, or wounding with intent to cause grievous bodily harm) while psychotic, by quantification of the broadband resonance signal from 31-phosphorus neurospectroscopy scans.

\section{Materials and methods}

Cerebral 31-phosphorus magnetic resonance spectroscopy was carried out in 15 male patients with schizophrenia who had seriously and violently offended (homicide, attempted murder, or wounding with intent to cause grievous bodily harm) while psychotic and in 12 age- and sex-matched normal control subjects. Data were obtained using a 1.5 T Marconi Eclipse system with a birdcage quadrature head coil dual-tuned to proton $(64 \mathrm{MHz})$ and 31P $(26 \mathrm{MHz})$. T1-weighted magnetic resonance images were acquired for spectral localization. Spectra were obtained using an image-selected in vivo spectroscopy sequence ( $\mathrm{TR}=10 \mathrm{~s} ; 64$ signal averages) localized on a $70 \times 70 \times 70 \mathrm{~mm}^{3}$ voxel.

${ }^{4}$ Head of Forensic Neurosciences, Lipid Neuroscience Group, Imperia College London and Three Bridges Medium Secure Unit, West London Mental Health NHS Trust, Middlesex, UK

\section{Results}

There was no significant difference in the broad resonances between the two groups, with the mean (standard error) percentage broadband signal for the patients being 57.8 (5.6) and that for the control subjects 57.7 (6.0). The phosphomonoesters and phosphodiesters narrow signals also did not differ between the groups.

\section{Conclusions}

Our data suggest that the membrane phospholipid hypothesis of schizophrenia may not apply to the subgroup of schizophrenia patients who have seriously and violently offended.

\section{Acknowledgements}

We thank the Three Bridges Medium Secure Unit and the MRC.

\section{Author details}

${ }^{1}$ Imaging Sciences Department, Hammersmith Hospital, Imperial College London, UK. ${ }^{2}$ Department of Radiology, University of California, San Diego, School of Medicine, San Diego, CA, USA. ${ }^{3}$ Three Bridges Medium Secure Unit, West London Mental Health NHS Trust, Middlesex, UK. ${ }^{4}$ Head of Forensic Neurosciences, Lipid Neuroscience Group, Imperial College London and Three Bridges Medium Secure Unit, West London Mental Health NHS Trust, Middlesex, UK.

Published: 22 April 2010

doi:10.1186/1744-859X-9-S1-S153

Cite this article as: Puri et al:: Brain cell membrane motion-restricted phospholipids in patients with schizophrenia who have seriously and dangerously violently offended. Annals of General Psychiatry 2010 9(Suppl 1):S153. 